\title{
A New Architecture Based on IoT and Machine Learning Paradigms in Photovoltaic Systems to Nowcast Output Energy
}

\author{
Guillermo Almonacid-Olleros ${ }^{1}$, Gabino Almonacid ${ }^{1, *}$, Juan Ignacio Fernandez-Carrasco ${ }^{1}$, \\ Macarena Espinilla-Estevez ${ }^{2}$ and Javier Medina-Quero ${ }^{2}$ \\ 1 Department of Electronic Engineering, Campus Las Lagunillas, 23071 Jaén, Spain; \\ gao00003@red.ujaen.es (G.A.-O.); jifernan@ujaen.es (J.I.F.-C.) \\ 2 Department of Computer Science, Campus Las Lagunillas, 23071 Jaén, Spain; mestevez@ujaen.es (M.E.-E.); \\ jmquero@ujaen.es (J.M.-Q.) \\ * Correspondence: galmona@ujaen.es
}

Received: 30 June 2020; Accepted: 26 July 2020; Published: 29 July 2020

\begin{abstract}
The classic models used to predict the behavior of photovoltaic systems, which are based on the physical process of the solar cell, are limited to defining the analytical equation to obtain its electrical parameter. In this paper, we evaluate several machine learning models to nowcast the behavior and energy production of a photovoltaic (PV) system in conjunction with ambient data provided by IoT environmental devices. We have evaluated the estimation of output power generation by human-crafted features with multiple temporal windows and deep learning approaches to obtain comparative results regarding the analytical models of PV systems in terms of error metrics and learning time. The ambient data and ground truth of energy production have been collected in a photovoltaic system with IoT capabilities developed within the Opera Digital Platform under the UniVer Project, which has been deployed for 20 years in the Campus of the University of Jaén (Spain). Machine learning models offer improved results compared with the state-of-the-art analytical model, with significant differences in learning time and performance. The use of multiple temporal windows is shown as a suitable tool for modeling temporal features to improve performance.
\end{abstract}

Keywords: photovoltaic systems; nowcasting energy generation; temporal windows

\section{Introduction}

Currently, photovoltaic (PV) power generation has been shown to be a successful technology with a remarkable level of maturity with more than $500 \mathrm{GW}$ of solar photovoltaic (PV) power installed all over the world at the end of 2018, in some cases running for several years, and with a forecast of 1 TW of total power being generated by 2022, most of it in large PV plants. The management of the operation and maintenance $(\mathrm{O} \& \mathrm{M})$ of these systems is a relevant research field for the solar PV industry [1,2].

Data represent a key asset in this PV management area, since they enable us to model the standard behavior of the system and to monitor its performance compared with the expected output determined by the model. This monitoring, when applied promptly and comprehensively, taking account of all the factors that may impact performance, enables early damage and fault detection, which then allows operation and maintenance actions to maximize the up-time and efficiency of PV plants.

Traditionally, approximate analytical expressions based on the physical laws and the electrical parameters of the solar cells, together with the engineering data of the devices that conform the PV system, have been used to build standard performance models. Leveraging the latest software advances in machine learning, a different approach can be taken by using regressors to build models, which learn from data on the actual behavior of the system during a relevant period of time and use 
the time series prediction to monitor performance. Machine learning approaches bring the advantage of modeling independently from the deployment and configuration parameters of the PV system, which are strongly affected by location and environmental conditions.

This work presents an important extension of the proposal [3], where two deep learning models showed a better performance in forecasting energy generation with regard to standard analytical models $[4,5]$. The main contribution of this work is evaluating in further detail the capabilities of data-driven models for nowcasting the energy generation of photovoltaic systems from ambient sensor information. In this way, two main data-driven approaches are evaluated: (i) human-crafted features which are computed by means of multiple temporal windows and (ii) deep learning models with automatic feature extraction and learning. Several configurations of segmentation and aggregation by means of temporal windows have been proposed showing an improvement in terms of performance and learning time. So, an important advance is made in this knowledge area through the use of machine learning techniques to make predictions about PV system consumption in order to check its status. In addition, an IoT module which collects photovoltaic data in real time within the Opera platform is described. The module has collected the evaluation data over 24 weeks, which are openly available to the scientific community.

The remainder of the paper is organized as follows: in Section 2, we detail the review of works related to our proposal; Section 3 describes the supporting infrastructure and IoT module for collecting real-time data within the Opera Digital Platform; Section 4 presents the methodology to develop data-driven nowcasting of PV system consumption; Section 5 introduces the results of the dataset collected by the Opera Digital Platform. Finally, conclusions and ongoing works are discussed in Section 6.

\section{Related Works}

PV systems are now considered a well-established technology for energy generation and have reached a significant maturity level. However, being relatively recent most of the systems have been running no more than 20 years $[1,6]$-means that there is not much experience in Operations and Maintenance $(\mathrm{O} \& \mathrm{M})$. Most of the tasks and tools regarding O\&M make little use of new information technologies such as big data, deep learning, business intelligence, etc. [7]. Up to now, the most common way to estimate the behavior of PV systems has been the use of classic models based on the physical process of the solar cell to define the analytical equation to obtain its electrical parameter [8]. There are many of these models with very different approaches, difficulty levels and results [4,9-11]. The main objective of these tools is to nowcast the electrical energy generated by the cells and also by the PV system.

Among all of these classic models, we have selected the Araujo model plus constant FF (FF: Fill Factor is a noteworthy solar cell figure regarding maximum power delivered vs. maximum current and maximum voltage of the cell; its upper limit is 1;) [4] to compare and evaluate the performance of PV systems with the performance estimated by our proposed machine learning model. Araujo is a standard PV model that combines enough accuracy with a very simple formulation $[4,8]$; additionally, it needs only a few variables to be measured: current and voltage of the cell, irradiation and ambient temperature [5].

Nevertheless, to obtain output energy using any of these classic models it is necessary to know a large number of parameters and specifications of the PV generator in question: technical specs, topology of the generator, location etc. One of the main advantages of our machine learning-based proposal is the ability to nowcast all of these parameters and specs independently, and hence enabling easier and more efficient PV deployment and customization.

Recently, several works regarding the use of new technologies to monitor and nowcast PV system behavior have been presented. However, none of them have been used in or have produced-a usable O\&M management system [7,12-14]. A previous work related to a O\&M analytics platform was presented in [15]. The use of new information technologies in O\&M management in the renewables sector has, up to now, been restricted to a few large and expensive platforms developed by companies to use in utility-scale generator power plants $[16,17]$. 
Regarding the collection of operating data to monitor PV systems, it is traditionally carried out with wired sensor data acquisition systems, which are sometimes expensive, allow little flexibility and have limited cloud connectivity. Recently, several works on the new concept of using IoT connectivity in monitoring the behavior of PV systems have been presented [12,14,18]. Incorporating these sensors in a comprehensive O\&M management tool has allowed us to develop a highly versatile and easy-operation data collection system with wireless sensors, which offers great advantages as regards ease of use, cost efficiency and standardization of data capture $[13,19,20]$.

Several proposals based on the IoT paradigm in photovoltaic systems have been presented in the relevant literature. In [21], a literature review of IoT energy platforms aimed at end users is presented, where platform selection, new energy platform construction and, finally, platform comparison are considered. In [22], the design and implementation of an IoT-based solar monitoring system for city-wide, large-scale, and distributed solar facilities in smart cities was presented. In [23], a solar tracking system enabling increased efficiency of photovoltaic systems was proposed. The proposed system executes a tracking algorithm in the Firebase web service and allows the exchange of data with said service through a NodeMCU development board, which has an integrated Wi-Fi module. Finally, in [24], the use of IoT and machine learning paradigms for next-generation solar power plant monitoring systems was analyzed and discussed.

Regarding the use of IoT and machine learning paradigms for analyzing sensor data streams, there are techniques that have proven to be successful in other contexts. For example, evaluation of single and multiple windows to segment and fuse temporal information from sensor data streams [25,26], whose window size can be imbalanced $[27,28]$ to aggregate data from shorter to longer terms, enriching the features of sensor streams.

On the other hand, the use of Deep Learning in temporal series has become a prolific research field [29]. Mainly, with the use of Long-Short Term Memory (LSTM) [30], which is a type of recurrent neural network that includes a memory and is designed to learn from sequence data, such as sequences of observations over time. LSTM is most widely used in natural language processing and speech recognition, can model temporal dependence between observations [31] and is suitable for prediction from sensor data [32]. LSTM has obtained encouraging results in several fields, such as activity recognition [28] or estimating building energy consumption [33]. Moreover, modeling spatial features in time series by means of Convolutional Neural Networks (CNNs) [31] qiu2017learning has achieved promising results in speech recognition [34] or gas classification [35], together with LSTM models [36].

\section{IoT Module for Real-Time Data Collection in the Opera Digital Platform}

In this section, we describe the IoT module for collecting the photovoltaic data in the Opera Digital Platform, which have been collected to nowcast output energy generation in the photovoltaic system.

Opera Project is a digital platform developed by an interdisciplinary team, covering the areas of ICTs, PV and Electronic Technology, and has been designed to provide O\&M management services for renewable energy installations [15]. This digital platform has been developed with the knowledge and the working data of the UniVer Project. This project see Figure 1 is a standard, medium-sized, grid-connected PV system that has been running for the last 20 years in the Campus of the University of Jaén [37]. The PV modules are made of 60 multicrystalline Si solar cells with $18.34 \%$ efficiency and a $156.75 \times 156.75 \mathrm{~mm}^{2}$ surface. The PV generator is composed of 220 of these modules with a topology of 20 (serial) $\times 11$ (parallel) and a total power of $59.4 \mathrm{~kW}$ at Standard Test Conditions (STCs; that is, $1000 \mathrm{~W} / \mathrm{m}^{2}$ of normal irradiance onto cells, cell temperature of $25^{\circ} \mathrm{C}$ and AM1.5 solar spectrum). 

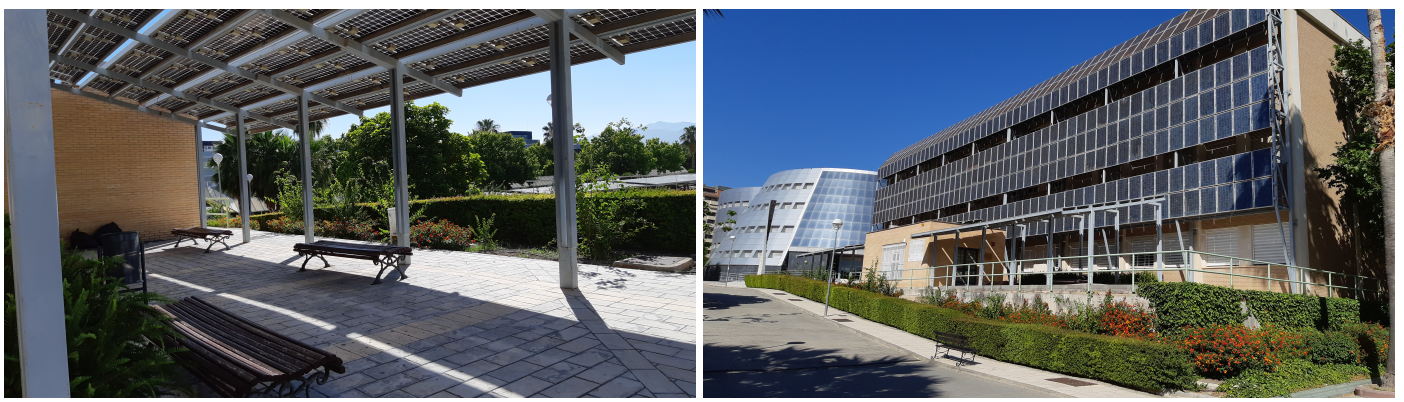

Figure 1. Two views of the photovoltaic (PV) generator of the UniVer Project. (a) PV pergola with semitransparent modules; (b) East view of the PV facade.

The Opera Platform is now also managing the O\&M of this PV system.

The main objective of the IoT-based PV system O\&M optimization module, besides reducing costs, is to monitor the generated energy. Energy $E_{T}$ is the end product of every electric generator and is computed as the integral of instantaneous power $P$ in a period of time $\mathrm{T}: E_{T}=\int_{T} P \cdot d t$. Electric power output is the instantaneous variable to be measured by this data collection system and also targeted by the models to nowcast the behavior of PV systems, such as the one developed in this paper. This output mainly relies upon the entry product: solar irradiance $G$ whose magnitude is defined by the square density of power incident on a surface measured in Watts per square meter $\left(\mathrm{W} / \mathrm{m}^{2}\right)$. The temperature and the specs of the PV generator (PVG) are the other inputs for this data collection system monitoring the performance of the PVG.

Monitoring of the PVG must be done following the European Standard IEC 61724 [38]. In line with this, the variables that have been measured are shown in Table 1. From these measured data and with the nominal specs of the PVG at STC, we compute derived parameters and metrics regarding losses in energy performance, which is useful to evaluate the behavior of the PV system and very helpful for fault diagnosis and descriptive operation analysis, such as : (i) global irradiation on the PVG surface, (ii) net energy from the PVG in a period of time, (iii) performance ratio and (iv) yields and losses. All of them are well defined analytically and conceptually in [38] and their function, meaning and usefulness are also described in [39-41]. In this work, we have focused on the nowcasting of output power generation, which is straightforwardly related to the analytical metrics on the behavior of the PV system.

In order to collect environmental and energy generation information from the Opera Digital Platform in real time, we have developed and deployed a genuine integration of ambient and power supply sensors. This is composed of a set of sensors based on IoT technology connections and controlled by a microprocessor which uploads the data by wireless network. These sensors measure the working data of the PVG and the environmental variables shown in Table 1, needed to monitor and nowcast PVG operation in accordance with standard [42].

Table 1. Variables measured by the data collection system.

\begin{tabular}{lll}
\hline Parameter & Symbol & Unit \\
\hline Irradiance on PV surface & $G_{I}$ & $\mathrm{~W} \cdot \mathrm{m}^{-2}$ \\
Ambient temperature & $T_{a m}$ & ${ }^{\circ} \mathrm{C}$ \\
PVG output current & $I_{A}$ & $\mathrm{~A}$ \\
PVG output voltage & $V_{A}$ & $\mathrm{~V}$ \\
PVG output power generation & $P_{A}$ & $\mathrm{~W}$ \\
\hline
\end{tabular}

The central unit of the IoT module is an Arduino. It is a standard board device that includes, in addition to a $\mu P$, an input data conditioner, a communication network interface and other display interfaces. The IoT module is responsible for collecting the photovoltaic data to send the information 
to the cloud by means of an internet connection (i.e.: wired, WiFi or modem). The module is powered by standard power supply or by solar panel plus battery.

The ambient sensors connected to the Arduino board detect: (i) solar irradiance, (ii) module temperature and (iii) ambient temperature. The irradiance sensor is a calibrated Si solar cell (calibration certificate from CIEMAT, the Spanish Research Centre in Energy, Environment and Tech.), with an analogical output from $0-5 \mathrm{~V}$ corresponding to an irradiance range from 0 to $1250 \mathrm{~W} / \mathrm{m}^{2}$. The ambient and cells temperature sensors are 4-wire Pt100 Probes, also with an analogical output of $0-5 \mathrm{~V}$, corresponding to a temperature range of -20 to $130{ }^{\circ} \mathrm{C}$. These two sensors, plus the corresponding interface circuitry, are included in a commercial unit made by Atersa S.L. (www.atersa.com), as we describe in Figure 2. The ambient sensors are placed close to the panels and are powered by their own solar mini-module. The ambient sensors send the measured data to the Arduino microprocessor using Zigbee protocol to enable direct wireless communication between the devices and the Arduino board [43] in open areas, which is inherent in the deployment of photovoltaic systems. We included the Zigbee connection since experimental results with other popular wireless technologies, such as Wi-Fi and Bluetooth, show that it is more energy efficient [44].

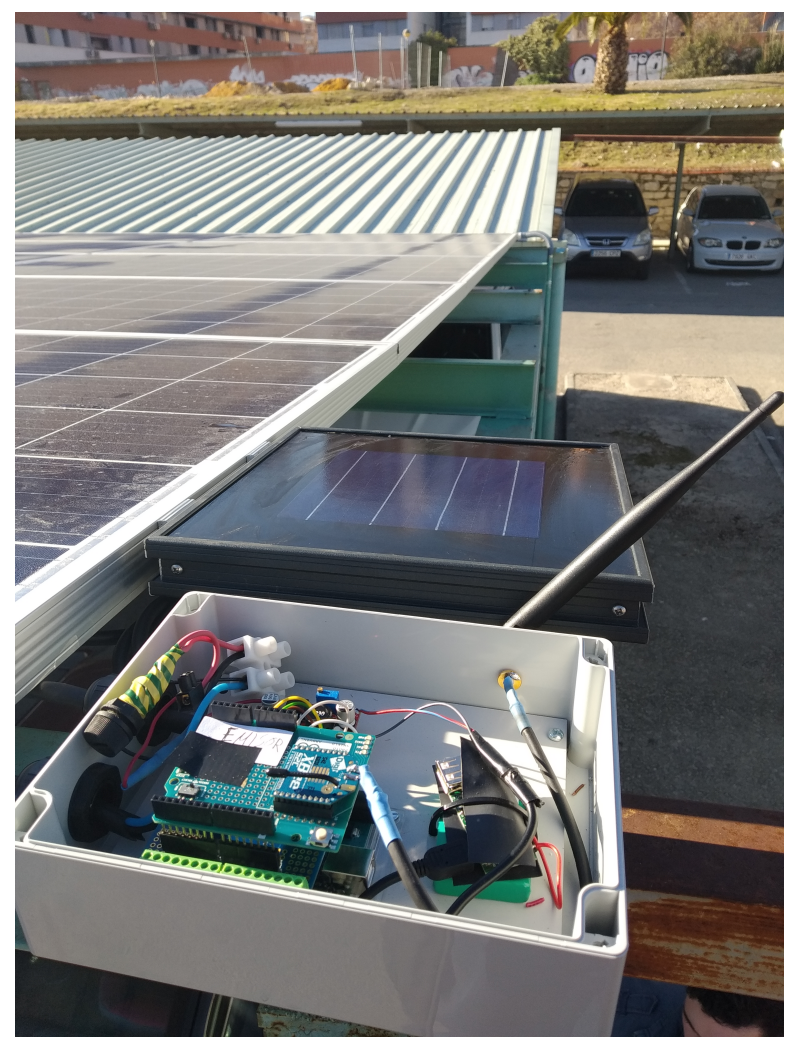

Figure 2. Radiation and temperature sensors unit.

The PVG data measured by the IoT module are the instantaneous values of output voltage and intensity, which enable the computing of output power by multiplying output voltage and current intensity. This is possible since the data are instantaneous values; in this case, the output of the PVG is DC current, so this way to obtain power is also valid for mean values over a period of time. Alternatively, an output power sensor can be installed, such as a power meter or a grid analyzer, to get some redundancy in the measured data and, with the second device, some additional secondary electrical output parameters.

Finally, in Figure 3 we show the voltage and current sensors, along with the microprocessor unit used to measure operation data of the UniVer Project PV generator. Figure 4 shows a schematic diagram of the data collection architecture. 

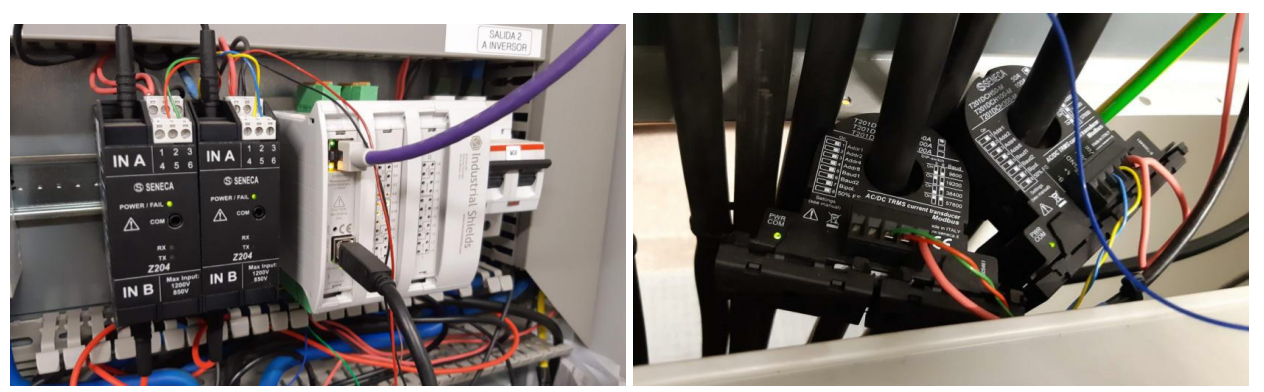

Figure 3. Current and voltage sensors and microprocessor unit. (a) Two voltage sensor devices for the two branch of the generator under study (black units) and the $\mu P$ unit (white one); (b) Two current sensor, toroidal cores, for the two branch of the generator under study.

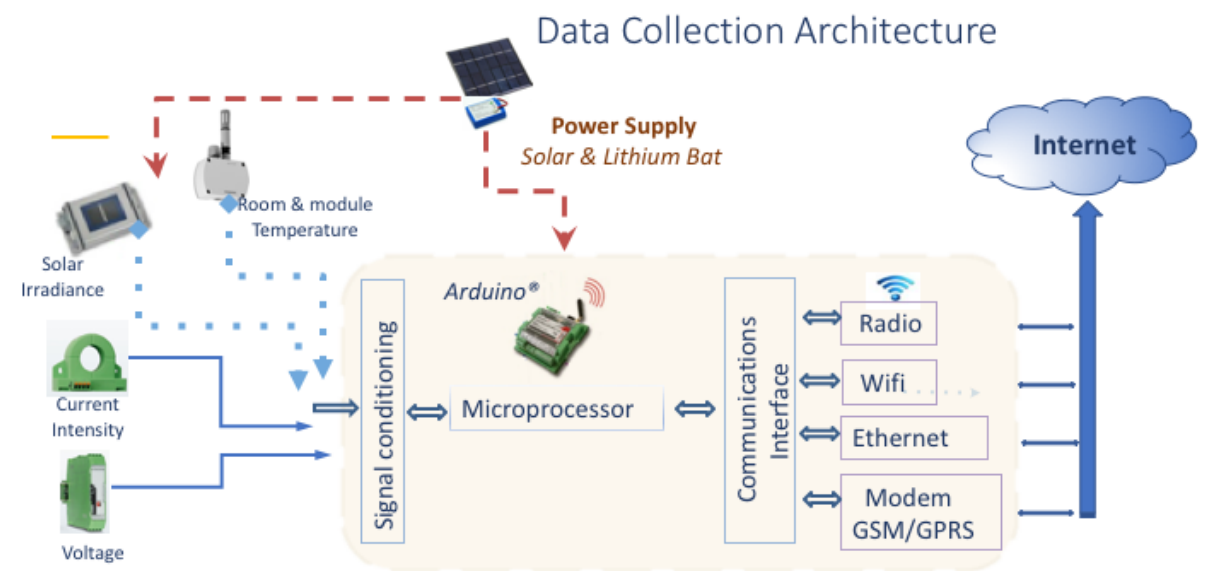

Figure 4. Architecture of the IoT module for collecting data in real-time.

\section{Machine Learning Approaches to Nowcast Power Generation}

In this section, we describe the methodology used for processing, segmenting and modeling the sensor data from the Opera PV System in order to nowcast output power generation from the ambient sensor information in real time.

As stated previously, several models are evaluated in this work. They are mainly grouped into: (i) human-crafted features and multiple temporal windows and (ii) deep learning for automatic feature extraction and learning. In the following sections, we detail: (first) basic segmentation with temporal sliding windows for sensor streams in a data-driven model; (second) modeling for human-crafted features and multiple temporal windows; and (third) deep learning approaches to nowcast output power generation of the Opera PV System.

\subsection{Data-Driven Model to Nowcast Power Generation}

Following a formal definition, a sensor $s$ collects data in real time in the form of a pair $\overline{s_{i}}=\left\{s_{i}, t_{i}\right\}$, where $s_{i}$ represents a given measurement and $t_{i}$ the time-stamp, respectively. Thus, the data stream of the sensor source $s$ is defined by $\overline{S_{s}}=\left\{\overline{s_{0}}, \ldots, \overline{s_{i}}\right\}$ and a given value in a timestamp $t_{i}$ by $S_{s}\left(t_{i}\right)=s_{i}$. In this work, irradiance on PV surface $G_{I}$, ambient temperature $T_{a m}$, PVG output $I_{A}$, PVG output voltage $V_{A}$ and PVG output power generation $P_{A}$ provide five data streams which describe the behavior and energy production of the PV system.

Next, temporal sliding windows, which are defined by the window size of a time interval $W_{w}=\left[W_{w}^{-}, W_{w}^{+}\right][45]$, segment the samples of a sensor stream $\overline{S_{s}}$ and aggregate the values $\overline{s_{i}}$ by a given aggregation function $T_{t}\left(S_{s}, W_{w}, t^{*}\right)$ : 


$$
T_{t}\left(S_{s}, W_{w}, t^{*}\right)=\bigcup_{s_{i}}^{\overline{s_{i}}} s_{i}, t_{i} \in\left[t^{*}-W_{w}^{-}, t^{*}-W_{w}^{+}\right]
$$

whose value of aggregation defines a given feature $T_{t}$ of the sensors $S_{s}$ in a current time $t^{*}$. In Figure 5 , we describe the segmentation and aggregation by temporal sliding windows in some visual examples of data streams.

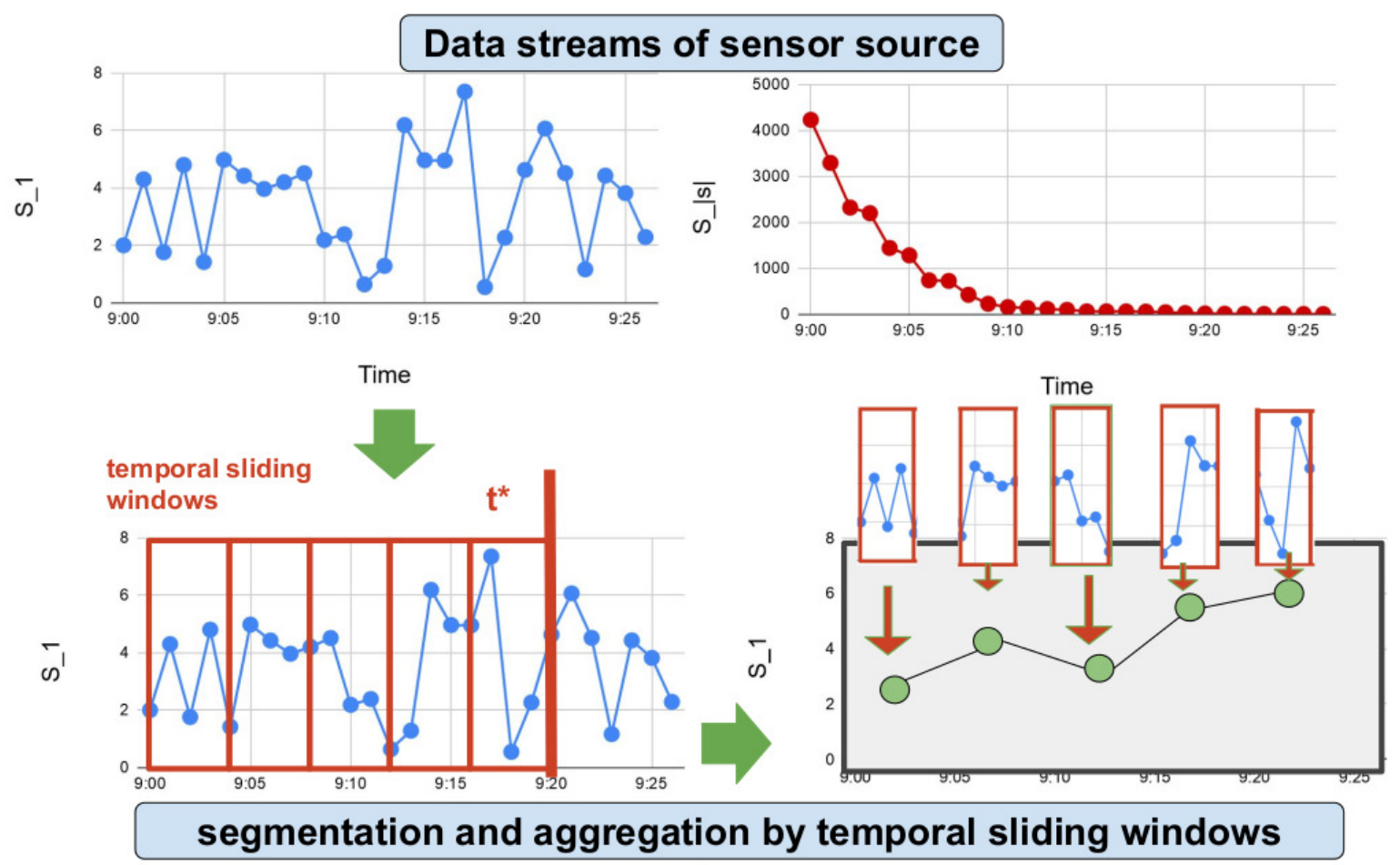

Figure 5. Example of data streams from sensor sources, segmentation and aggregation by temporal sliding windows.

\subsection{Human-Crafted Features and Multiple Temporal Windows for Efficient Nowcasting of Output Power Generation}

In this section, we describe human-crafted features based on multiple sliding temporal windows where an expert defines an aggregation function to process sensor streams training a data-driven regressor to compute a feature vector for learning purposes.

Among the broad spectrum of models, we focus on efficient regressor, which enables both learning and evaluating on micro boards in real time under fog computing environments [27]. To this end, we evaluate a human-crafted feature approach [46], where the aggregation functions and multiple windows of different sizes are defined by experts. In concrete terms, we include the following configuration of models:

- Aggregation functions $T_{t}$ based on statistical metrics, such as maximal, minimal, average and standard deviation have been defined in this configuration as they have been demonstrated as relevant features in describing sensor streams [47].

- Segmentation and fusion of temporal information from sensor streams with: (i) single window, (ii) multiple windows [25], and (iii) incremental windows [27] to aggregate data from shorter to longer terms enriching the features of sensor streams. Window size is also defined by human criteria.

- Classification from efficient regressors, with low learning time and training requirements, such as linear regression, k-nearest neighbors $(\mathrm{kNN})$, support vector machines (SVM) and random forest (RF). 
Therefore, starting from a set of input sensors $S=\left\{S_{1}, \ldots, S_{S}, \ldots, S_{|S|}\right\}$, a set of window sizes $W=\left\{W_{1}, \ldots, W_{w}, \ldots, W_{|W|}\right\}$ and a set of aggregation functions $T=\left\{T_{1}, \ldots, T_{t}, \ldots, T_{|T|}\right\}$ we define a total number of features $|S| \times|W| \times|T|$ which describe the sensor streams $S$ for each point of time $t^{*}$ [47]. Since our model is based on a data-driven supervised approach, the features which describe the sensor streams are associated for each point of time $t^{*}$ with a target sensor to nowcast $S^{*}$ (not included in the input sensors $\left.S \cap S^{*}=\right)$ :

$$
T_{1}\left(S_{1}, W_{1}, t^{*}\right), \ldots, T_{t}\left(S_{S}, W_{w}, t^{*}\right), \ldots T_{|T|}\left(S_{|S|}, W_{|W|}, t^{*}\right) \rightarrow S^{*}\left(t^{*}\right)
$$

\subsection{Deep Learning Modeling to Nowcast Output Power Generation}

In this section, we describe DL models to nowcast output power generation in a PV device. Contrary to the previous proposal, DL does not require human-crafted features and data pre-processing is applied to compute a homogeneous sequence of data between the different collection rates from raw sensor sources. Here, a minimal signal segmentation is defined by sliding temporal windows of short-term window size, which is related to a minimal temporal granularity $\Delta$. The raw data are averaged $U=\mu$ for each short-term temporal window within the segment.

So, we obtain a sequence of data for each sensor source, whose sequence size is the same for all sources $S_{S}$ :

$$
S^{*}\left(t^{*}\right) \rightarrow\left\{\begin{array}{l}
\mu\left(S_{1},[0, \Delta], t^{*}\right) \rightarrow \mu\left(S_{1},[\Delta, 2 \Delta], t^{*}\right), \ldots, \mu\left(S_{1},[\Delta|W|-\Delta, \Delta|W|], t^{*}\right) \\
\ldots \\
\mu\left(S_{|S|},[0, \Delta], t^{*}\right) \rightarrow \mu\left(S_{|S|},[\Delta, 2 \Delta], t^{*}\right), \ldots, \mu\left(S_{|S|},[\Delta|W-1|, \Delta|W|], t^{*}\right)
\end{array}\right.
$$

which are related to the target sensor to nowcast $S^{*}$ for each current time $t^{*}$ under a sliding window approach.

Once the input and output data from the DL model are defined, in this work, we propose two architectures of DL neural networks to nowcast the output power generation of the PV device, which have been shown as suitable configurations to sequence time series in sliding window approaches [48].

- $\quad$ 2LSTM. Two layers of LSTM which have been previously identified as a suitable configuration to nowcast energy load [49].

- $\quad 3 C N N+2 L S T M$. Three layers of CNN are firstly integrated as spatial feature extractors. Next, two layers of LSTM model the temporal dependencies from CNN. The combination of CNN-LSTM hybrid networks has been selected due to providing encouraging results in modeling output power generation [50].

In Table 2, we include the parameters and layers for each proposed model.

Table 2. Configurations of Convolutional Neural Networks.

\begin{tabular}{ll}
\hline 2LSTM & 3CNN+2LSTM \\
\hline LSTM (32 units) & 2 kernels $\times 16$ filters \\
dropout $(0.25)$ & Re-Lu \\
LSTM (32 units) & 2 kernels $\times 32$ filters \\
dropout $(0.25)$ & Re-Lu \\
connected (1 unit) & 2 kernels $\times 64$ filters \\
activation function: Re-Lu & Re-Lu \\
loss function: MAE & dropout $(0.25)$ \\
& LSTM (32 units) \\
& dropout $(0.25)$ \\
& LSTM (32 units) \\
& dropout $(0.25)$ \\
& connected (1 unit) \\
& activation function: Re-Lu \\
& loss function: MAE \\
\hline
\end{tabular}




\section{Evaluation}

In this section, we present the evaluation of our proposal. First we shall present the experimental setup, then the results obtained and, finally, we will discuss our proposal based on the results presented.

\subsection{Experimental Setup}

In this section, we describe the experimental setup and results of a case study developed in the University of Jaén (Spain), where the Opera Project and PV device were deployed. The IoT module which collected the photovoltaic data in real time within the Opera platform was running from the 9th of June to the 23rd of November 2019, generating data collection over 168 days. The location of the IoT module in the campus of the University of Jaén was (latitude: 37.787253 , longitude: -3.776258).

In the experimental setup, five sensors, which were installed in the PV device, collected the following measures: irradiance, ambient temperature, module temperature, output current and output voltage, as described in Section 3. The output power generation to be estimated by the machine learning model was obtained using output current and output voltage according to the following equation: $P=V \dot{I}$.

Both data and learning models are openly available to the scientific community at this GitHub repository: https://github.com/galmonacid/opera/. Below, we detail the configuration and results in nowcasting output power generation by several machine learning models.

- Human-crafted features and multiple temporal windows. We evaluate the nowcasting performance of the following models with human-crafted features and multiple temporal windows and times with the configurations shown below:

- $\quad$ Linear regression, with intercept $=$ True.

- $\quad$ kNN (k-Nearest Neighbors), with number of neighbours $=5$.

- SVM (Support Vector Machine), with kernel = polynomial .

- $\quad$ Random forest, with minimum samples leaf $=1$ and minimum samples split $=2$.

For each of these four models, three sliding temporal window configurations were defined and evaluated:

- $\quad T=10 \mathrm{~min}$, one single 10-min temporal window.

- $\quad T=30 \mathrm{~min}$, three 10-min temporal windows.

- $\quad T=90 \mathrm{~min}$, three incremental temporal windows, with a 10-min, 20-min and 60-min window.

- Deep Learning approaches, where we evaluate the performance and learning time of two DL models: 2LSTM and 3CNN+2LSTM, as described in Section 4.3. Concretely, we have evaluated two segmentation configurations: $10 \min \Delta=10 \mathrm{~m}$ and $5 \min \Delta=5 \mathrm{~m}$ :

- $\Delta=10$ m defined by a 90-minute sequence of data whose sequence length is $|W|=9, W=$ $\{[0 \mathrm{~m}, 9 \mathrm{~m}],[10 \mathrm{~m}, 19 \mathrm{~m}], \ldots,[80 \mathrm{~m}, 89 \mathrm{~m}]\}$. For $\Delta=10 \mathrm{~m}$ and $|W|=18 \mathrm{~W}=\{[0 \mathrm{~m}, 4 \mathrm{~m}],[5 \mathrm{~m}$, $9 \mathrm{~m}], \ldots,[85 \mathrm{~m}, 89 \mathrm{~m}]\}$ for $\Delta=5 \mathrm{~m}$. This configuration generated a total of 24,031 samples for learning purposes.

- $\Delta=5 \mathrm{~m}$, defined by a 90-minute sequence of data whose sequence length is $|W|=18$, $|W|=18 W=\{[0 \mathrm{~m}, 4 \mathrm{~m}],[5 \mathrm{~m}, 9 \mathrm{~m}], \ldots,[85 \mathrm{~m}, 89 \mathrm{~m}]\}$ for $\Delta=5 \mathrm{~m}$. This configuration generated a total of 48,062 samples for learning purposes.

In order to nowcast output power generation from the ambient data collected in the PVS, we compared the predicted and ground truth in the tests using 30 -fold cross validation. We note the ambient data from photovoltaic sources has been normalized using the max-min method in a previous learning stage. 


\subsection{Results}

In this section, we describe the obtained results from the standard analytical method and the machine learning approaches described in the work.

Output power generation was collected by the IoT module representing the ground truth for evaluation purposes. The estimated output power generation for each model was based on data from ambient sensors. The prediction versus the ground truth for the full time-line of tests were compared using Mean Absolute Error (MAE), Root Mean Squared Error (RMSE) and coefficient of determination (R2). With the 30-fold cross validation configuration, we also computed learning time and evaluation time to assess the resource consumption of the models.

First, we evaluated the Araujo model which provides a base performance provided by the standard analytical method. The results from this baseline model are shown in Table 3:

Table 3. Araujo error metrics.

\begin{tabular}{rrrr}
\hline Model & RMSE (W) & MAE (W) & R2 \\
\hline Araujo & 641.36 & 354.81 & 0.9947 \\
\hline
\end{tabular}

Second, we evaluated one of the data-driven approaches analyzed in this work: regressor models which nowcast energy generation by means of human-crafted features computed from sensor streams. The results are shown in Table 4 in terms of RMSE, MAE and R2 metrics.

Table 4. Error metrics of human-crafted feature models with different sliding windows approaches.

\begin{tabular}{lrrrr}
\hline Model & Sliding Window Sizes & RMSE (W) & MAE (W) & R2 \\
\hline \multirow{3}{*}{ Linear Regression } & $10 \mathrm{~min}$ & 637.20 & 425.74 & 0.9948 \\
& $10 \mathrm{~min}+10 \mathrm{~min}+10 \mathrm{~min}$ & 590.27 & 375.11 & 0.9955 \\
& $10 \mathrm{~min}+20 \mathrm{~min}+60 \mathrm{~min}$ & 537.37 & 323.04 & 0.9963 \\
\hline \multirow{3}{*}{ kNN } & $10 \mathrm{~min}$ & 466.76 & 229.62 & 0.9972 \\
& $10 \mathrm{~min}+10 \mathrm{~min}+10 \mathrm{~min}$ & 536.11 & 249.29 & 0.9963 \\
& $10 \mathrm{~min}+20 \mathrm{~min}+60 \mathrm{~min}$ & 528.40 & 253.13 & 0.9964 \\
\multirow{3}{*}{ Random Forest } & $10 \mathrm{~min}+10 \mathrm{~min}+10 \mathrm{~min}$ & 375.49 & 183.49 & 0.9982 \\
& $\mathbf{1 0} \mathbf{m i n}+\mathbf{2 0} \mathbf{~ m i n}+\mathbf{6 0} \mathbf{~ m i n}$ & 360.13 & $\mathbf{1 7 3 . 4 7}$ & $\mathbf{0 . 9 9 8 3}$ \\
\hline \multirow{3}{*}{ SVM } & $10 \mathrm{~min}$ & 4474.17 & 2794.36 & 0.7421 \\
& $10 \mathrm{~min}+10 \mathrm{~min}+10 \mathrm{~min}$ & 4593.69 & 2835.49 & 0.7281 \\
& $10 \mathrm{~min}+20 \mathrm{~min}+60 \mathrm{~min}$ & 4410.17 & 2653.65 & 0.7493 \\
\hline
\end{tabular}

Furthermore, in order to evaluate the computational energy consumption, we have included a comparison of learning and evaluation time for the models based on human-crafted features in Table 5 .

Third, we evaluated the data-driven approach based on deep learning. To compare the results with Araujo and models based on human-crafted features, we provide the comparison of the performance of DL models in terms of RMSE, MAE and R2 metrics in Table 6 and the learning and evaluation time in Table 7.

Finally, as summary of the results of the different models, in Table 8 we include a comparison between the different approaches: Araujo, the best-performing DL model (3CNN+2LSTM) and the best regressor among human-crafted feature approaches (random forest $90 \mathrm{~min}$ ).

In order to provide a visual representation of the nowcasting of energy consumption, in Figure 6 we show a 2-day sample test comparing measured output power generation with the regressor models. 
Table 5. Human-crafted feature models time metrics.

\begin{tabular}{lrrr}
\hline Model & Sliding Window Sizes & Learning Time (ms) & Evaluation Time (ms) \\
\hline \multirow{3}{*}{ Linear Regression } & $10 \mathrm{~min}$ & 11.46 & 2.10 \\
& $10 \mathrm{~min}+10 \mathrm{~min}+10 \mathrm{~min}$ & 36.67 & 2.72 \\
& $10 \mathrm{~min}+20 \mathrm{~min}+60 \mathrm{~min}$ & 37.25 & 2.81 \\
\hline \multirow{3}{*}{$\mathrm{kNN}$} & $10 \mathrm{~min}$ & 86.52 & 13.00 \\
& $10 \mathrm{~min}+10 \mathrm{~min}+10 \mathrm{~min}$ & 213.55 & 51.50 \\
& $10 \mathrm{~min}+20 \mathrm{~min}+60 \mathrm{~min}$ & 196.54 & 52.11 \\
\multirow{3}{*}{ Random Forest } & $10 \mathrm{~min}+10 \mathrm{~min}+10 \mathrm{~min}$ & $22,743.35$ & 42.89 \\
& $10 \mathrm{~min}+20 \mathrm{~min}+60 \mathrm{~min}$ & $69,790.30$ & 45.54 \\
& & $73,499.40$ & 42.03 \\
\multirow{3}{*}{ SVM } & $10 \mathrm{~min}+10 \mathrm{~min}+10 \mathrm{~min}$ & $30,912.46$ & 322.88 \\
& $10 \mathrm{~min}+20 \mathrm{~min}+60 \mathrm{~min}$ & $48,459.79$ & 846.61 \\
& & $49,373.02$ & 857.88 \\
\hline
\end{tabular}

Table 6. Error metrics of Deep Learning approaches based on Long Short-Term Memory (LSTM) and Convolutional Neural Network (CNN)+LSTM.

\begin{tabular}{lrrrr}
\hline Model & Segmentation & RMSE (W) & MAE (W) & R2 \\
\hline \multirow{2}{*}{ 2LSTM } & $5 \mathrm{~min}$ & 2393.75 & 618.70 & 0.9262 \\
& $10 \mathrm{~min}$ & 706.57 & 376.76 & 0.9936 \\
\hline \multirow{2}{*}{$3 \mathrm{CNN}+2 \mathrm{LSTM}$} & $5 \mathrm{~min}$ & 2384.14 & 583.11 & 0.9271 \\
& $10 \mathrm{~min}$ & 531.08 & 274.87 & 0.9964 \\
\hline
\end{tabular}

Table 7. Deep Learning models time metrics.

\begin{tabular}{lrrr}
\hline Model & Segmentation & Learning Time (ms) & Evaluation Time (ms) \\
\hline 2LSTM & $10 \mathrm{~min}$ & $222,657.12$ & 6951.65 \\
3CNN+2LSTM & $10 \mathrm{~min}$ & $197,593.52$ & 5627.01 \\
\hline
\end{tabular}

Table 8. Summary of error metrics for best configurations of human-crafted features and DL approaches.

\begin{tabular}{lrrr}
\hline Model & RMSE (W) & MAE (W) & R2 \\
\hline Araujo & 641.36 & 354.81 & 0.9947 \\
3CNN+2LSTM & 531.08 & 274.87 & 0.9964 \\
Random Forest & 360.13 & 173.47 & 0.9983 \\
\hline
\end{tabular}

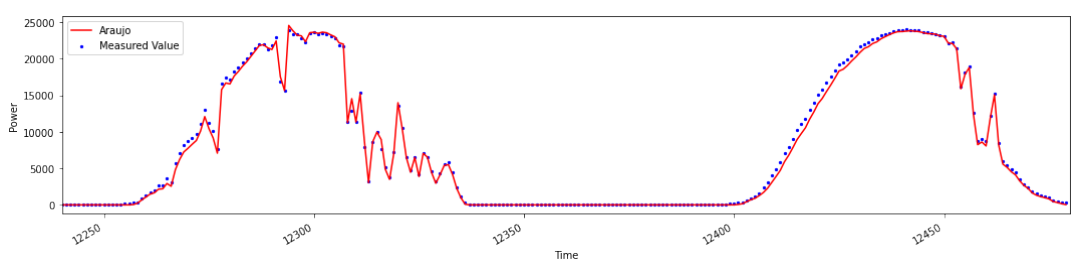

(i)

Figure 6. Cont. 


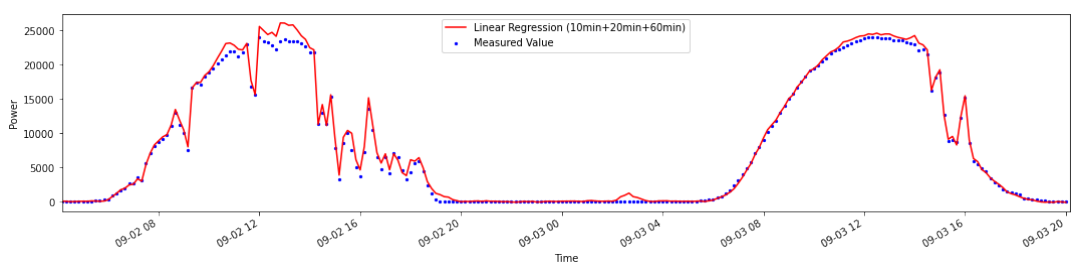

(ii)

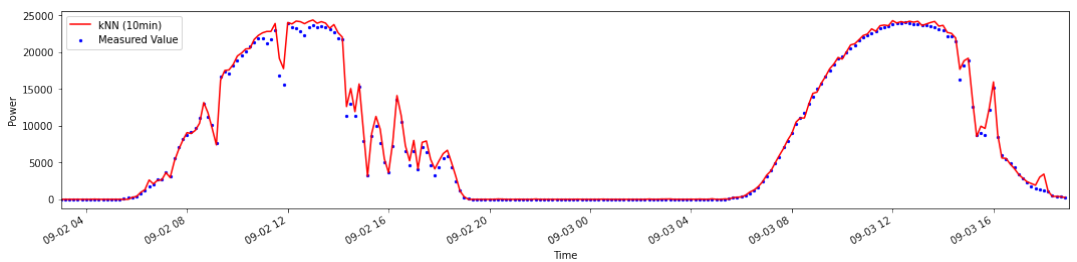

(iii)

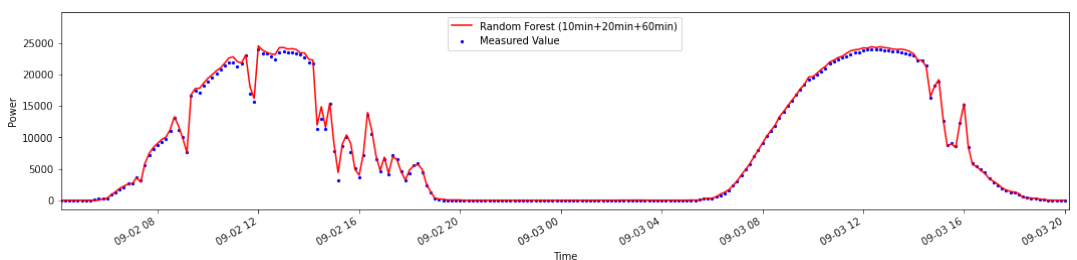

(iv)

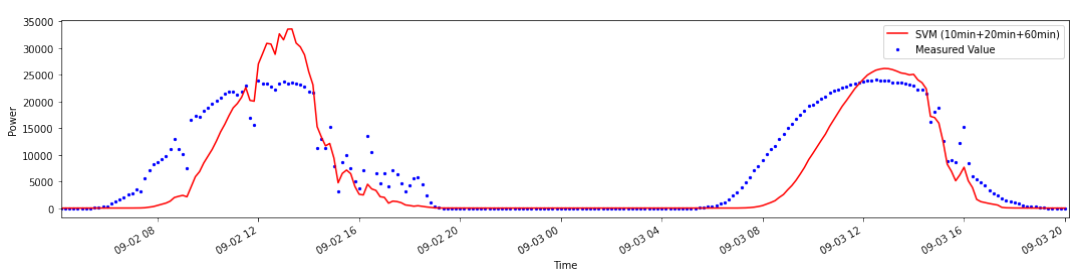

(v)

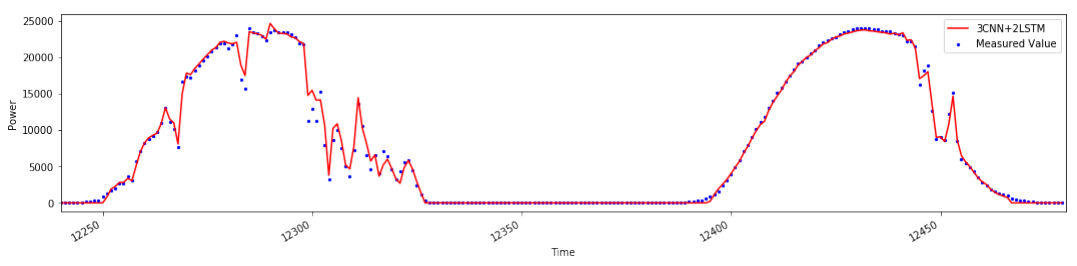

(vi)

Figure 6. We show 2-day samples of ground truth of output power generation compared with the predictions. From the top to bottom: (i) the Araujo model, (ii) linear regression, (iii) kNN, (iv) random forest, (v) SVM, (vi) 3CNN+2LSTM.

\subsection{Discussion}

In this work we describe an IoT module for collecting ambient sensor information and output energy consumption from the photovoltaic system deployed under the Opera Project. In order to evaluate the standard behavior of the system and to monitor its performance, we have focused on nowcasting output energy generation from the ambient sensor devices. To this end, two different approaches for machine learning models have been proposed: (i) human-crafted features and multiple temporal windows and (ii) deep learning for automatic feature extraction and learning.

Both approaches present encouraging performance in nowcasting output energy generation in the photovoltaic system based on data collected from ambient sensors; however, we highlight 
the model based on human-crafted features and multiple temporal windows for its lower learning time and best results. Specifically, we note: (i) the use of multiple imbalanced temporal windows increases nowcasting performance, (ii) random forest is the best regressor and (iii) kNN provides an excellent balance between learning time and results. Moreover, the use of kNN should be highly recommended for nowcasting energy generation in photovoltaic systems using fog-based approaches, where mini boards could perform the data learning in a short time using low computational resources and computational energy consumption.

In the case of DL approaches, the use of CNN+LSTM provides improved nowcasting performance when comparing the results with the Araujo analytical model. This fact is due to the automatic feature extraction generated by $\mathrm{CNN}$, which summarizes the key patterns to nowcast output power generation, providing a remarkable improvement compared with only using LSTM. The performance of the DL model with 10-min segmentation increases compared to 5-min segmentation because short-term segmentation duplicates the number of input variables in the sequence of samples and the higher complexity of data reduces nowcasting performance. However, the human-crafted features model with imbalanced temporal windows has overtaken the performance of DL approaches and the Araujo analytical model, coming out as the leading model according to the results presented in this work.

\section{Conclusions and Ongoing Works}

In this work, an IoT module and data-driven models to nowcast output energy generation integrated in the Opera Digital Platform project have been described. The IoT module is based on Arduino and low-cost sensors which collect ambient and energy data sources in a photovoltaic system. The IoT module has collected the data presented in this work over 24 weeks.

Two approaches based on machine learning have been evaluated: (i) human-crafted features with multiple temporal windows, and (ii) deep learning models. CNN+LSTM, kNN and random forest provide better performance compared with the standard analytical model Araujo. In the case of CNN+LSTM, the advantage of DL is the lack of human intervention in feature definition. The performance of kNN is remarkable, with notably low learning time and providing fog integration capabilities in micro boards. Finally, random forest with incremental temporal windows had the highest performance in terms of error metrics.

A potential advance in this line of work would consist of an in-depth analysis of the diagnosis, typology and fail patterns in PV systems to predict these events by means of machine learning models.

Author Contributions: conceptualization, G.A.-O., G.A. and J.I.F.-C.; machine learning model, G.A.-O., M.E.-E. and J.M.-Q.; hardware, sensor, J.I.F.-C.; validation, formal analysis, investigation, supervision, all authors. All authors have read and agreed to the published version of the manuscript.

Funding: This contribution has been supported by the Cátedra ELAND for Renewable Energies of the University of Jaén and by the Spanish government by means of the project RTI2018-098979-A-I00 and the Action 1 (2019-2020) no. EI_TIC01 of the University of Jaén.

Conflicts of Interest: The authors declare no conflict of interest.

\section{Abbreviations}

The following abbreviations are used in this manuscript:

FF Fill Factor

IoT Internet of Things

LD linear dichroism

CNN Convolutional Neural Network

LSTM Long Short-Term Memory

DL Deep Learning

O\&M Operation and Maintenance 
PV Photovoltaic

PVG PV Generator

PVS PV Systems

STC Standard test conditions for solar cells

\section{References}

1. Europe, S.P. Global Market Outlook For Solar Power/2019-2023; Technical Report; Solar Power Europe: Brussels, Belgium, 2019.

2. Silvestre, S.; Silva, M.A.D.; Chouder, A.; Guasch, D.; Karatepe, E. New procedure for fault detection in grid connected PV systems based on the evaluation of current and voltage indicators. Energy Convers. Manag. 2014, 86, 241-249. [CrossRef]

3. Almonacid-Olleros, G.; Almonacid, G.; Fernandez-Carrasco, J.; Quero, J.M.Q. Opera. DL: Deep Learning Modelling for Photovoltaic System Monitoring. Multidiscip. Digit. Publ. Inst. Proc. 2019, 31, 50.

4. Fuentes, M.; Nofuentes, G.; Aguilera, J.; Talavera, D.; Castro, M. Application and validation of algebraic methods to predict the behaviour of crystalline silicon PV modules in Mediterranean climates. Sol. Energy 2007, 81, 1396-1408. [CrossRef]

5. Araujo, G.; Sanchez, E. Analytical expressions for the determination of the maximum power point and the fill factor of a solar cell. Sol. Cells 1982, 5, 377-386. [CrossRef]

6. Europe, S.P. OEM Best Practice Guidelines; Technical Report; Solar Power Europe: Brussels, Belgium, 2016.

7. Bermejo, J.F.; Fernández, J.F.G.; Polo, F.O.; Márquez, A.C. A Review of the Use of Artificial Neural Networks Models for Energy and Reliability Prediction. A Study for the Solar PV, Hydraulic and Wind Energy Sources. Appl. Sci. 2019, 9, 1844. [CrossRef]

8. Luque, A.; Hegedus, S. Handbook of Photovoltaic Science and Engineering; John Wiley \& Sons: Hoboken, NJ, USA, 2011.

9. Rus-Casas, C.; Aguilar, J.; Rodrigo, P.; Almonacid, F.; Pérez-Higueras, P. Classification of methods for annual energy harvesting calculations of photovoltaic generators. Energy Convers. Manag. 2014, 78, 527-536. [CrossRef]

10. Almeida, M.P.; Muñoz, M.; de la Parra, I.; Perpiñán, O. Comparative study of PV power forecast using parametric and nonparametric PV models. Sol. Energy 2017, 155, 854-866. [CrossRef]

11. De la Parra, I.; Muñoz, M.; Lorenzo, E.; García, M.; Marcos, J.; Martínez-Moreno, F. PV performance modelling: A review in the light of quality assurance for large PV plants. Renew. Sustain. Energy Rev. 2017, 78, 780-797. [CrossRef]

12. Daliento, S.; Chouder, A.; Guerriero, P.; Pavan, A.M.; Mellit, A.; Moeini, R.; Tricoli, P. Monitoring, diagnosis, and power forecasting for photovoltaic fields: A review. Int. J. Photoenergy 2017, 2017. [CrossRef]

13. Raza, M.; Aslam, N.; Le-Minh, H.; Hussain, S.; Cao, Y.; Khan, N.M. A critical analysis of research potential, challenges, and future directives in industrial wireless sensor networks. IEEE Commun. Surv. Tutor. 2017, 20,39-95. [CrossRef]

14. Fuentes, M.; Vivar, M.; Burgos, J.M.; Aguilera, J.; Vacas, J.A. Design of an accurate, low-cost autonomous data logger for PV system monitoring using Arduino ${ }^{\mathrm{TM}}$ that complies with IEC standards. Sol. Energy Mater. Sol. Cells 2014, 130. [CrossRef]

15. Almonacid-Olleros, G.; Vidal, P.; Fernández-Carrasco, J.; Almonacid, G. Opera Project: Analytic platform based on Big Data and Business Intelligence to improve Operation and Maintenance in PV generators. In Proceedings of the IEEE International Conference on Environment and Electrical Engineering, Milan, Italy, 12-14 March 2018.

16. Greenpower. 2019. Available online: https://www.enelgreenpower.com/es/historias/a/2017/08/BigData-el-oro-digital-de-las-renovables (accessed on 28 July 2020).

17. Acciona. Renewable Energy Control Center (cecoer). 2019. Available online: https://www.acciona.com/ es/lineas-de-negocio/energia/proyectos-emblematicos/centro-control-energias-renovables/ (accessed on 28 July 2020).

18. Kumar, N.M.; Atluri, K.; Palaparthi, S. Internet of Things (IoT) in Photovoltaic Systems. In Proceedings of the 2018 National Power Engineering Conference (NPEC), Madurai, India, 9-10 March 2018. 
19. Jayaprakash, M.; Kavitha, D.; Ramkumar, M.S.; Balachander, K.; Krishnan, M.S. Achieving efficient and secure data acquisition for cloud-supported internet of things in grid connected solar, wind and battery systems. Math. Comput. For. Nat. Resour. Sci. 2019, 11, 144-155.

20. Spanias, A.S. Solar energy management as an Internet of Things (IoT) application. In Proceedings of the 2017 8th International Conference on Information, Intelligence, Systems \& Applications (IISA), Larnaca, Cyprus, 28-30 August 2017.

21. Martín-Lopo, M.M.; Boal, J.; Sánchez-Miralles, Á. A literature review of IoT energy platforms aimed at end users. Comput. Netw. 2020, 171, 107101. [CrossRef]

22. Shapsough, S.; Takrouri, M.; Dhaouadi, R.; Zualkernan, I. An IoT-based remote IV tracing system for analysis of city-wide solar power facilities. Sustain. Cities Soc. 2020, 57, 102041. [CrossRef]

23. Gutierrez, S.; Rodrigo, P.M.; Alvarez, J.; Acero, A.; Montoya, A. Development and Testing of a Single-Axis Photovoltaic Sun Tracker through the Internet of Things. Energies 2020, 13, 2547. [CrossRef]

24. Karbhari, G.; Nema, P. Iot \& machine learning paradigm for next generation solar power plant monitoring system. Int. J. Adv. Sci. Technol. 2020, 29, 6894-6902.

25. Banos, O.; Galvez, J.M.; Damas, M.; Guillen, A.; Herrera, L.J.; Pomares, H.; Rojas, I.; Villalonga, C.; Hong, C.S.; Lee, S. Multiwindow fusion for wearable activity recognition. In International Work-Conference on Artificial Neural Networks; Springer: Berlin/Heidelberg, Germany, 2015; pp. 290-297.

26. Espinilla, M.; Medina, J.; Hallberg, J.; Nugent, C. A new approach based on temporal sub-windows for online sensor-based activity recognition. J. Ambient Intell. Humaniz. Comput. 2018, 1-13. [CrossRef]

27. López Medina, M.Á.; Espinilla, M.; Paggeti, C.; Medina Quero, J. Activity Recognition for IoT Devices Using Fuzzy Spatio-Temporal Features as Environmental Sensor Fusion. Sensors 2019, 19, 3512. [CrossRef]

28. Medina-Quero, J.; Zhang, S.; Nugent, C.; Espinilla, M. Ensemble classifier of long short-term memory with fuzzy temporal windows on binary sensors for activity recognition. Expert Syst. Appl. 2018, 114, 441-453. [CrossRef]

29. Gamboa, J.C.B. Deep learning for time-series analysis. arXiv 2017, arXiv:1701.01887.

30. Hochreiter, S.; Schmidhuber, J. LSTM can solve hard long time lag problems. In Advances in Neural Information Processing Systems; MIT Press: Cambridge, MA, USA, 1997; pp. 473-479.

31. Wang, J.; Chen, Y.; Hao, S.; Peng, X.; Hu, L. Deep learning for sensor-based activity recognition: A survey. Pattern Recognit. Lett. 2019, 119, 3-11. [CrossRef]

32. Quero, J.M.; Medina, M.Á.L.; Hidalgo, A.S.; Espinilla, M. Predicting the urgency demand of copd patients from environmental sensors within smart cities with high-environmental sensitivity. IEEE Access 2018, 6, 25081-25089. [CrossRef]

33. Mocanu, E.; Nguyen, P.H.; Gibescu, M.; Kling, W.L. Deep learning for estimating building energy consumption. Sustain. Energy Grids Netw. 2016, 6, 91-99. [CrossRef]

34. Abdel-Hamid, O.; Mohamed, A.R.; Jiang, H.; Deng, L.; Penn, G.; Yu, D. Convolutional neural networks for speech recognition. IEEE/ACM Trans. Audio Speech Lang. Process. 2014, 22, 1533-1545. [CrossRef]

35. Peng, P.; Zhao, X.; Pan, X.; Ye, W. Gas classification using deep convolutional neural networks. Sensors 2018. [CrossRef]

36. Huang, C.J.; Kuo, P.H. A deep cnn-lstm model for particulate matter (PM2. 5) forecasting in smart cities. Sensors 2018, 18, 2220. [CrossRef]

37. Drif, M.; Pérez, P.; Aguilera, J.; Almonacid, G.; Gomez, P.; De la Casa, J.; Aguilar, J. Univer Project. A grid connected photovoltaic system of 200kWp at Jaén University. Overview and performance analysis. Sol. Energy Mater. Sol. Cells 2007, 91, 670-683. [CrossRef]

38. IEC. European Standard IEC 61724-1. In Photovoltaic Systems Performance-Part 1 Monitoring; International Electrotechnical Commission: Geneva, Switzerland, 2017.

39. Klise, K.A.; Stein, J.S.; Cunningham, J. Application of IEC 61724 Standards to Analyze PV System Performance in Different Climates. In Proceedings of the 2017 IEEE 44th Photovoltaic Specialist Conference (PVSC), Washington, DC, USA, 25-30 June 2017.

40. Blaesser, G; Munro, D. Guidelines for the Assessment of Photovoltaic Plants; European Commision: Brussels, Belgium, 1995; Volume C, pp. 1-38.

41. Blaesser, G.; Zaaiman, W. On-Site Power Measurements on Large PV Arrays. In Tenth EC Photovoltaic Solar Energy Conference; Springer: Dordrecht, The Netherlands, 1991. 
42. European Standard IEC 61724:2017. Photovoltaic system performance monitoring. In Guidelines for Measurement, Data Exchange and Analysis; International Electrotechnical Commission: Geneva, Switzerland, 2017.

43. Ferreira, H.G.C.; Canedo, E.D.; De Sousa, R.T. IoT architecture to enable intercommunication through REST API and UPnP using IP, ZigBee and arduino. In Proceedings of the 2013 IEEE 9th International Conference on Wireless and Mobile Computing, Networking and Communications (WiMob), Lyon, France, 7-9 October 2013; pp. 53-60.

44. Siekkinen, M.; Hiienkari, M.; Nurminen, J.K.; Nieminen, J. How low energy is bluetooth low energy? comparative measurements with zigbee/802.15. 4. In Proceedings of the 2012 IEEE Wireless Communications and Networking Conference Workshops (WCNCW), Paris, France, 1 April 2012; pp. 232-237.

45. Banos, O.; Galvez, J.M.; Damas, M.; Pomares, H.; Rojas, I. Window size impact in human activity recognition. Sensors 2014, 14, 6474-6499. [CrossRef]

46. Cruciani, F.; Vafeiadis, A.; Nugent, C.; Cleland, I.; McCullagh, P.; Votis, K.; Giakoumis, D.; Tzovaras, D.; Chen, L.; Hamzaoui, R. Comparing CNN and Human Crafted Features for Human Activity Recognition. In Proceedings of the 2019 IEEE SmartWorld, Ubiquitous Intelligence \& Computing, Advanced \& Trusted Computing, Scalable Computing \& Communications, Cloud \& Big Data Computing, Internet of People and Smart City Innovation (SmartWorld/SCALCOM/UIC/ATC/CBDCom/IOP/SCI), Leicester, UK, 19-23 August 2019.

47. Espinilla, M.; Medina, J.; Salguero, A.; Irvine, N.; Donnelly, M.; Cleland, I.; Nugent, C. Human Activity Recognition from the Acceleration Data of a Wearable Device. Which Features Are More Relevant by Activities? Multidiscip. Digit. Publ. Inst. Proc. 2018, 2, 1242. [CrossRef]

48. Selvin, S.; Vinayakumar, R.; Gopalakrishnan, E.; Menon, V.K.; Soman, K. Stock price prediction using LSTM, RNN and CNN-sliding window model. In Proceedings of the 2017 International Conference on Advances in Computing, Communications and Informatics (ICACCI), Udupi, India, 13-16 September 2017; pp. 1643-1647.

49. Marino, D.L.; Amarasinghe, K.; Manic, M. Building energy load forecasting using deep neural networks. In Proceedings of the IECON 2016 - 42nd Annual Conference of the IEEE Industrial Electronics Society, Florence, Italy, 24-27 October 2016; pp. 7046-7051.

50. Kim, T.Y.; Cho, S.B. Predicting the household power consumption using CNN-LSTM hybrid networks. In Proceedings of the International Conference on Intelligent Data Engineering and Automated Learning, Madrid, Spain, 21-23 November 2018; pp. 481-490.

Sample Availability: The data collected by the photovoltaic system and code of the models from Opera Project are available in https://github.com/galmonacid/opera/.

(C) 2020 by the authors. Licensee MDPI, Basel, Switzerland. This article is an open access article distributed under the terms and conditions of the Creative Commons Attribution (CC BY) license (http:/ / creativecommons.org/licenses/by/4.0/). 